\section{Planning to use science}

Vera Rich looks at the Soviet Union's recently published Five Year Plan, in which the prominence usually accorded to science and technology is again apparent.

A $S$ EXPECTED, the Soviet Union's Five Plan for the period 1976-1980, soon to be submitted to the party congress, gives top priority to greater efficiency throughout the economy, specifically in agriculture and industry. But the framework of the Plan as a whole considerably curtails the almost rhetorical aims for science also expressed by the Plannamely, "the further expansion and deepening of investigations of the laws of nature and society, increasing its contribution to the solution of problems of current importance in the construction of the material and technological basis of communism, accelerating scientific and technical progress and the growth of effectiveness of production, increasing the wellbeing and culture of the nation, and shaping the communist outlook of the workers".

The Plan's modest targets for growth, investment and production seem to have been set deliberately low to take into account possible set-backs of the sort that hampered the previous Plan, such as crop failures and inefficiency. The target for national income growth, at $24-28 \%$, is lower than the $28 \%$ achieved by the 1971-1975 Plan. Industrial production will rise $35-39 \%$ as against $43 \%$ previously, while the growth of investment in agriculture is to fall from $60 \%$ to $30 \%$. Under the general slogan of improved "quality" and increased production, it is demanded that $90 \%$ of industrial growth and all the growth in agriculture and production is to be met by higher labour productivity (as opposed to $80 \%$ in the previous plan).

Within this general prospect of harder and better work with no encouragement of major initiatives, which is seen by many as the design of ageing politicians whose active lifetime is unlikely to run to the full term of the Plan, the scientific projects selected for specific mention and encouragement form an interesting crosssection of research. At one end the emphasis on pure and applied mathematics is related in the Plan to the need for the more efficient and extensive computerisation of the economy - growth in the computer and automated cquipment sections, at $60-80 \%$, far outstrips the anticipated average for the industrial sector as a whole, and there are calls for cuts in the share of manual labour in production
At the other end there is the use of space research for the study of natural resources, meteorology and navigation, and for "other needs of the national economy". Nuclear, plasma, solid state and low temperature physics also receive special mention, as do quantum electronics, optics and astronomy; the need for new materials is stressed, especially magnetic semiconductors, superconductors, and "technically valuable" crystals, and the whole physics programme is grouped in the Plan with the need to develop atomic power and other new sources of energy. Indeed, energy-selected industries, including the chemical and petrochemical sectors, will be among the fastest-growing. The 1980 oil target is up $27 \%$, and a rise of $42 \%$ in natural gas will enhance the country's potential as an energy exporter.

In a similar manner, the biology programme is linked both to the need to develop new crops and improvements in occupational health. The main aims for agriculture include the development of animal husbandry and the fodder base, but there will be strong pressures for an improvement in agricultural productivity.

To a certain extent, such elaborations of the purposes of research projects in a time of financial cut-backs may be considered as a justification for their continuation. But the problem with Soviet science, which for all the latitude its practitioners possess operates within a rigid system of state planning, is more complex. With only limited resources of labour and capital available, certain projects-the space programme, for example-acquire a favoured position over others as a result of a complex decision-making process in which potential defence applications or international prestige can be important.

Apart from enjoying considerable material benefits, scientists working in these areas usually find few obstructions in the pursuit of their work. It is possible, for example, to order equipment from abroad; and the procedures for obtaining experimental materials are considerably facilitated. As a result, these fields inevitably attract a great majority of the most gifted talent. On the other hand, scientists not working in a "favoured" field find themselves at a considerable disadvantage, at least with respect to the bureaucracy: requisitions for experimental materials must be made far in advance and of ten can only be fulfilled by an unofficial exchange of surpluses between laboratories or institutions.

The gap between favoured and nonfavoured projects and institutes tends to widen as a result, and once a particular subject, say space research, has acquired a measure of prestige, a major cut-back is more difficult to order, at least in the political life-time of those who first sanctioned it. As for the non-favoured projects, these tend to be staffed by the relatively less able, resulting in a relative decline in performance, so that in certain fields, like precision instruments, a technological short-fall emerges which makes the purchase of required equipment abroad more likely.

Accordingly, although throughout the Soviet Union's history the presumption has been that Soviet science must inevitably outstrip that of the west, the day of total independence of Soviet technology still lies in the future. This is tacitly admitted by the present plan, which includes the purchase from the west of equipment for producing mineral fertilizers and for the development of the oil, gas, paper, cellulose, and "certain other" industries. The stress on the need for computerisation and automation suggests that there will be considerable (if unacknowledged) reliance on western know-how.

One possible solution open to the authorities is the purchase of foreign equipment which can then be studied and reproduced, thus saving research and development costs. The Plan's proposals may therefore be a disguised way of purchasing expertise. During the last few years the Soviet Union has signed a number of agreements on technological cooperation with Western countries which are clearly intended to be of hilateral benefit, and it will be interesting to observe how the fields of cooperation may change in the future as the Soviet Union closes the short-fall gap in this or that branch of technology.

The sensitive relationship between science and industry in the Soviet Union will continue to be revealed over the coming five years. In the short term, because the rigid targets imposed on industry often leave no time for re-tooling or re-equipping, the introduction of some cost-saving and efficient new process may be reflected in the quarterly returns as a fallingoff in various industries. The contribution of science to the long term aims of higher productivity and improved efficiency, to be effective, will demand better management and planning of techniques. Yet, oddly, this is a comparatively neglected area in the new Five Year Plan. 